\title{
AshwaMAX and Withaferin A inhibits gliomas in cellular and murine orthotopic models
}

\author{
Edwin Chang ${ }^{1,2,3,4}$, Christoph Pohling ${ }^{1,2,3,4}$, Arutselvan Natarajan ${ }^{1,2,3,4}$, Timothy H. \\ Witney ${ }^{1,2,3,4}$, Jasdeep Kaur ${ }^{1,2,3,4}$, Lingyun $\mathbf{X u}^{1,2,3,4}$, Gayatri Gowrishankar ${ }^{1,2,3,4}$, Aloma L

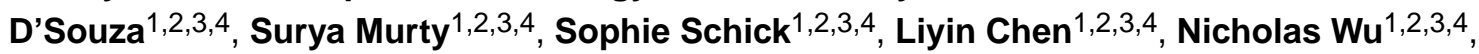 \\ Phoo Khaw ${ }^{1,2,3,4}$, Paul Mischel ${ }^{5}$, Taher Abbasi ${ }^{6}$, Shahabuddin Usmani ${ }^{6}$, Parag Mallick ${ }^{1,2,3,4}$, \\ and Sanjiv S. Gambhir $1,2,3,4$ \\ ${ }^{1}$ Departments of Radiology and Bioengineering, Stanford University, Palo Alto, CA, USA \\ ${ }^{2}$ Molecular Imaging Program at Stanford, Stanford University, Palo Alto, CA, USA \\ ${ }^{3}$ Canary Center at Stanford for Cancer Early Detection, Stanford University, Palo Alto, CA, USA \\ ${ }^{4}$ Nuclear Medicine, Stanford University, Palo Alto, CA, USA \\ ${ }^{5}$ Department of Medicine, Ludwig Institute for Cancer Research, University of California at San \\ Diego, La Jolla, California, USA \\ ${ }^{6}$ Cellworks Group Inc., San Jose, CA, USA
}

\section{Abstract}

Glioblastoma multiforme (GBM) is an aggressive, malignant cancer Johnson and O'Neill ( $\mathrm{J}$ Neurooncol 107: 359-364, 2012). An extract from the winter cherry plant (Withania somnifera), AshwaMAX, is concentrated (4.3\%) for Withaferin A; a steroidal lactone that inhibits cancer cells Vanden Berghe et al. (Cancer Epidemiol Biomark Prev 23: 1985-1996, 2014). We hypothesized that AshwaMAX could treat GBM and that bioluminescence imaging (BLI) could track oral therapy in orthotopic murine models of glioblastoma. Human parietal-cortical glioblastoma cells (GBM2, GBM39) were isolated from primary tumors while U87-MG was obtained commercially. GBM2 was transduced with lentiviral vectors that express Green Fluorescent Protein (GFP)/firefly luciferase fusion proteins. Mutational, expression and proliferative status of GBMs were studied. Intracranial xenografts of glioblastomas were grown in the right frontal regions of female, nude mice ( $\mathrm{n}=3-5$ per experiment). Tumor growth was followed through BLI. Neurosphere cultures (U87-MG, GBM2 and GBM39) were inhibited by AshwaMAX at $\mathrm{IC}_{50}$ of 1.4, 0.19 and $0.22 \mu \mathrm{M}$ equivalent respectively and by Withaferin $\mathrm{A}$ with $\mathrm{IC}_{50}$ of $0.31,0.28$ and $0.25 \mu \mathrm{M}$ respectively. Oral gavage, every other day, of AshwaMAX ( $40 \mathrm{mg} / \mathrm{kg}$ per day) significantly reduced

Correspondence to: Edwin Chang; Sanjiv S. Gambhir.

Electronic supplementary material The online version of this article (doi:10.1007/s11060-015-1972-1) contains supplementary material, which is available to authorized users.

Compliance with ethical standards

Conflict of interest The Authors declare no conflict of interests

Animal studies All preclinical studies were approved by the Institutional Animal Care and Use Committee of Stanford University. All ethical guidelines and standards were adhered to. 
bioluminescence signal ( $\mathrm{n}=3$ mice, $\mathrm{p}<0.02$, four parameter non-linear regression analysis) in preclinical models. After 30 days of treatment, bioluminescent signal increased suggesting onset of resistance. BLI signal for control, vehicle-treated mice increased and then plateaued. Bioluminescent imaging revealed diffuse growth of GBM2 xenografts. With AshwaMAX, GBM neurospheres collapsed at nanomolar concentrations. Oral treatment studies on murine models confirmed that AshwaMAX is effective against orthotopic GBM. AshwaMAX is thus a promising candidate for future clinical translation in patients with GBM.

\section{Keywords}

Glioblastoma; Bioluminescent; Preclinical; AshwaMAX; Withaferin A

\section{Introduction}

Glioblastoma multiforme (GBM) is a deadly form of malignant cancer with a median survival time of 15 months and a 5-year survival of $2.3 \%$ [1]. The moniker of "multiforme" reflects the heterogeneous morphological, molecular and genomic profile that the disease presents. Given the cancer's aggressive nature, current interventions consist of equally aggressive surgical resection and subsequent radio- and chemotherapy/immunotherapy. The cancer is heterogeneous, however and thus standardized, non-specific therapies are often ineffective [1]. To address the challenge of sub-optimal response, this new century has witnessed the emergence of classification strategies where cohorts of glioblastomas have been identified through novel molecular, genomic or molecular imaging biomarkers [3, 4]. For instance, gliomas can now be categorized as Classical, Mesenchymal, Proneural or Neural based upon the expression of certain molecular signatures such as wild-type and vIII variant Epidermal Growth Factor Receptor (EGFR), PDGF-AA and -BB, and Isocitrate Dehydrogenase I (IDH1) [5-8]. Classification of GBM based upon EGFR expression is particularly relevant as aggressiveness of glioblastomas has been associated with high EGFR expression [9].

Such developments have, in turn, spurred investigations into novel drugs that, in contrast to established, nonspecific therapies, are found by taking advantage of the new molecular classification protocols described above. In particular, new drugs have been found that interact with specific molecular targets or signaling pathways that are associated with the cancer class in question. For example, through this approach, the multi-histone deacetylase inhibitor, panobinostat was found to be therapeutically effective against diffuse pontine gliomas [10]. In addition, these searches have uncovered not only drugs that have been developed de novo but also drugs that were formerly targeted for other indications [11-13].

Withaferin A, an example of the latter, is a steroidal lactone that originates from the roots and leaves of the winter cherry plant (Withania somnifera or WS) and is the first member of the withanolides to be discovered [14]. Within the Ayurvedic and Unani medical tradition, the roots and leaves of the winter cherry plant (known as Ashwagandha or "scent of horse") are the raw material for numerous herbal medicines and are applied to a wide range of physical and mental health problems [15]. A unified insight into Withaferin A's action is 
still forthcoming, but current molecular and mechanistic studies into Withaferin A have shown it to be a downregulator of numerous transcription factors such as NF- $\mathrm{kB}$ and Sp1 $[16,17]$. It has been reported that the steroid also interacts with HSP70 and proteasomes [18]. Given such targets, it is not surprising that Withaferin A has been found to inhibit neovascularization via alterations in VEGF gene expression and calcium signaling [16, 19].

Such changes are often associated with altered EGFR signaling and therefore carcinogenesis, thus we were compelled to explore Withaferin A's (and by implication, Ashwagandha's) effect on glioblastoma cells. In addition, although Withaferin A has been studied in cell culture and in murine models for various types of cancers [20-24], to the best of our knowledge it has not been studied for GBM in living subjects. In this article, we report on how exposure to a specialized WS root extract (Trade Name: AshwaMAX), that contains $4.3 \%$ of Withaferin A, significantly impeded the growth of several glioblastoma cells both in culture and in pre-clinical xenograft models. Our study is the first to show the efficacy of AshwaMAX (and by implication, Withaferin A) against orthotopic xenograft models of human glioblastomas.

\section{Materials and methods}

\section{Purchase of chemical reagents}

Unless otherwise stated, all chemicals were purchased either from Selleckchem Inc.

(Houston, TX, USA) or Sigma-Aldrich (St. Louis, MO, USA).

\section{Preparation of Withaferin A and AshwaMAX}

Withaferin A was purchased in their purified form from Selleckchem (Houston, TX, USA). AshwaMAX, a root extract from the winter cherry tree, was supplied by Pharmanza Herbal Pvt Ltd. (Gujarat, India), and the extract was tested for the presence of Withaferin A [25]. The extract was a brown powder, standardized to contain no less than $4.3 \%$ Withaferin A with total Withanolides of $8.4 \%$ as determined by HPLC analysis (USP method).

\section{Cell culture studies}

Two patient-derived GBM lines (GBM2, GBM39) and one commercially available GBM cell line (U87-MG from ATCC, Manassas, VA, USA) were used for our studies. GBM2 originated from the Stanford University Medical Hospital and was obtained for research purposes after approval from the University's Internal Review Board. We gratefully acknowledge GBM39 as a gift from Dr. Paul Mischel (Ludwig Institute for Cancer Research, University of California at San Diego). These lines, along with U87 were used principally for cell culture experiments.

For all bioluminescence work, we used genetically-modified GBM2 and GBM39 whereby cells were transfected with lentiviral vectors that expressed either firefly luciferase (for GBM39) or a fusion protein of GFP and firefly luciferase (for GBM2) [26, 27]. Briefly, explant cultures of GBM2 and GBM 39 cells were transduced with HIV1-based lentiviral vectors expressing firefly luciferase (Luc) for GBM39, or a fusion protein of GFP and firefly luciferase (GFP/Luc) for GBM2. Lentiviral vectors were generated by transfection of $293 \mathrm{~T}$ 
cells with plasmids encoding the vesicular stomatitis virus $\mathrm{G}$ envelope, gagpol, and Fluc (generously provided by Dr. Y. Ikeda, Mayo Clinic, Rochester, MN) or with the GFP/Luc fusion protein (generously provided from Dr. Michelle Monje, Stanford University, Stanford, CA). Conditioned medium, containing viral vectors, was harvested $48 \mathrm{~h}$, posttransfection, filtered $(0.45 \mu \mathrm{m})$, and frozen until use. Cells were transduced using viral supernatants, and expression of luciferase was confirmed by measuring cellular luciferase activity (IVIS Spectrum; Xenogen Corporation, Alameda, CA). Luciferase-modified GBM cells were then collected and used to establish intracranial tumors that were monitored for drug response by longitudinal bioluminescence imaging. U87-MG was grown in DMEM (Life Technologies)/10 \% FBS/and 1× Penicillin/Steptocmycin (Life Technologies). GBM2 and GBM39 were grown in a defined, serum-free media of a 1:1 mixture of Neurobasal-A Medium (1 $\times$ /DMEM/F12(1 $\times$ ) that also contained HEPES Buffer Solution (10 mM), MEM Sodium Pyruvate Solution $1 \mathrm{mM}$, MEM Non-Essential Amino Acids Solution $10 \mathrm{mM}(1 \times)$, GlutaMAX-I Supplement (1×) and Antibiotic-Antimycotic (1×). All solutions are from Invitrogen/Life Technologies Inc. The full working media also contained h-EGF ( $20 \mathrm{ng} / \mathrm{ml})$, h-FGF-basic-154 (20 ng/ml), h-PDGF-AA (10 ng/ml), h-PDGF-BB (10 ng/ml) and Heparin Solution, $0.2 \%$ ( $2 \mathrm{ug} / \mathrm{ml})$ as growth factors (all from Shenandoah Inc.) and B-27 (Invitrogen/ Life Technologies) as supplements.

\section{Cellular readout assays}

To assess how our selected drugs affected cellular function, we established a number of readout assays.

Cellular proliferation-Cellular proliferation was assessed, indirectly, via the Alamar Blue assay. Intrinsic mitochondrial activity (and by implication, cellular proliferation) was assessed by measuring the oxidation of exogenously added Resaurin by the cell's internal mitochondrial respiratory complexes [28]. Briefly, cells were seeded onto 96-well plates (Corning, 5000 cells/well). $24 \mathrm{~h}$ after, escalating doses of drugs were added systematically to three wells per concentration. Final concentration of drugs ranged from 0.01 up to $200 \mu \mathrm{M}$. Alamar blue was added after two days and $24 \mathrm{~h}$ later, the plates were scanned on a Tecan 800 multi-channel fluorescent (Excitation: 560 (20) nm, Emission: 590 (10 nm) microplate reader.

Proliferation was measured directly via counting of trypsinized single cell suspensions on a standard haemocytometer (Fisher Scientific Inc., Fremont, CA) or on a Celigo S Automated Cytometer (Nexcelom Inc., Lawrence, MA).

Cell viability-The Celigo S Cellular Cytometer was also used to assess for occurrence of cell death by reading for percent of trypan blue positive cells (Nexcelom Inc., Lawrence, MA). Cell viability was determined by measuring for percentage of cells positive for propidium iodide $(1 \mu \mathrm{g} / \mathrm{ml})$ staining via standardized techniques [29]. An alternative approach was to run single cell suspensions that had been incubated with propidium iodide $(1 \mu \mathrm{g} / \mathrm{ml})$ though an ARIA (Becton-Dickinson Inc.) FACS sorter to identify positive (dead) versus negative (live) cells. 
Cellular apoptosis-Apoptotic index was determined by staining for Annexin V scoring for the amount of Annexin $\mathrm{V}$ positive cells as per a standardized protocol provided by eBiosciences Inc. The scoring was done either on a FACS/ARIA (BD Biosciences) or a CELIGO S cytometer counter from Nexcelom Inc.

\section{Western assays for EGFRvIII}

Western immune-assessment of wild type and vIII variant of EGFR was performed as per previously described protocols [26, 30]. Briefly, Antibodies used in this study for the detection of total EGFR, and phospho-EGFR (Y1080), were obtained from Cell Signaling; antibody for detection of h-actin was obtained from Sigma (St. Louis, MO).

\section{Preclinical studies}

Intracranial injection of patient-derived glioblastoma lines-All preclinical protocols have been approved by the Institutional Animal Care and Use Committee (IACUC) of Stanford University. The patient-derived human glioblastoma cells, GBM2 (p20-25) and GBM39 (P4-9) were injected with the use of a stereotactic instrument (Supplemental Figure S3) [31] into the right parietal region of the brain of female nu/nu mice (ages 6-10 weeks). Injection site was $2 \mathrm{~mm}$ to the right of the central suture and 0.5 $\mathrm{mm}$ anterior to the lambda junction. Depth of injection was 2-3 $\mathrm{mm}$.

Visualization of tumors: Growth of tumors was monitored either via bioluminescence imaging (BLI) or T1- and T2-weighted Magnetic Resonance Imaging (MRI) [32]. Luciferase activity was visualized by injecting mice intraperitoneally with $100 \mu \mathrm{l}$ of firefly luciferase $(30 \mathrm{mg} / \mathrm{ml})$ and the luminescence was monitored, 10 min later, by placing the mouse under nose-cone anesthesia, into an IVIS Spectrum (Xenogen Inc., Alameda, CA, USA). To visualize the soft tissue structure within tumors, the mice were subjected to MRI. The mice were injected intraperitoneally with $100 \mu \mathrm{l}$ of a gadolinium-containing contrast agent (Multihance, $0.1 \mathrm{~mol} / \mathrm{kg}$ mouse) to maximize the visualization of the tumors.

MRI was performed at the Stanford Clark Center Small Animal Imaging Facility in an actively-shielded Discovery MR901 General Electric 7T horizontal bore scanner (GE Healthcare) including Integrated Electronics Company (IECO) gradient drivers, an Agilent $120 \mathrm{~mm}$ inner diameter shielded gradient insert $(600 \mathrm{mT} / \mathrm{m}, 1000 \mathrm{~T} / \mathrm{m} / \mathrm{s})$, EXCITE2 electronics; the supporting LX11 platform; and a $3 \mathrm{~cm}$ inner diameter Millipede quadrature transmit/receive volume RF coil. Animals were anesthetized with $2 \%$ isoflurane in oxygen, and physiological monitoring included respiration, and temperature feedback for surface body temperature maintenance by warm airflow over the animal. A fast spoiled gradient echo $($ FSPGR $)$ sequence $\left(\mathrm{TR}=9.7 \mathrm{~ms} ; \mathrm{TE}=2.1 \mathrm{~ms}\right.$; flip angle $=5^{\circ} ; \mathrm{NEX}=20 ; \mathrm{FOV}=2$ $\mathrm{cm}$; image matrix $=160 \times 160$; slice thickness $=1 \mathrm{~mm}$ ) was used to acquire 3 sets of 12,12 , and 8 orthogonal T1-weighted images in the axial, sagittal, and coronal planes through the mass, respectively.

Oral Gavage of AshwaMAX: AshwaMAX was suspended in a solution of PBS to a stock concentration of $25-50 \mathrm{mg} / \mathrm{ml}$. The suspension was applied via oral gavage, every other day at an input of $40 \mathrm{mg}$ equivalence/kg mouse/day. Growth of tumors was tracked as described 
above with weekly or twice weekly scans of bioluminescence. BLI was done both before and after start of the administration of drug. Vehicle-treatment consisted of administering mice an equivalent volume of PBS via oral gavage.

Fluorescent and bioluminescent microscopy: To assess the morphology and microenvironment of the growing tumors, selected mice (control and/or therapy) were sacrificed and the brain excised and frozen in OCT. When appropriate, the brain was sliced into 10-30 um axial or sagittal sections via a Leica CM 1850 cryostat (Leica Biosystems, Richmond, USA) around the site of intracranial injection. The slices were adhered to glass slides and either direct fluorescent microscopy was performed (to detect GFP-positive tumor cells) in the presence of a cellular counter stain such as DAPI or the slides were fixed and stained for hemotoxylin and eosin via a standard protocol [33]. Bioluminescent microscopy was visualized under either an Olympus LV200 bioluminescent microscope (Olympus Life Sciences, Waltham, MA) or an IVIS Spectrum Imaging System (Xenogen Corporation, Alameda, CA). Bioluminescent microscopy by the LV200 was performed either at 100× or $400 \times$ magnification for acquisition times of 2 and $10 \mathrm{~min}$ respectively. In addition to incubation with D-luciferin $(30 \mathrm{mg} / \mathrm{ml})$, supplementary reagents of $5 \mathrm{mM}$ ATP, $2 \mathrm{mM}$ $\mathrm{MgSO}_{4}$ and $0.5 \mathrm{mM}$ Coenzyme A were added to the reaction mix.

\section{Statistical analysis}

All statistical analyses were performed either with Excel 2010 (Microsoft Corp.) or with PRISM v6.0 (Graph Pad Software, San Diego, CA) software. Univariate significance analysis of experimental data was determined by the Student $t$ test while multivariate analysis was determined through 2-way ANOVA. Differences were considered significant when $\mathrm{P}<0.05$. Data always reported as mean $\pm \mathrm{SD}$.

\section{Results}

\section{Cell culture studies}

\section{Morphology of GBM neurospheres and expression of EGFRvIll-Typical} glioblastoma (GBM) cell cultures formed neurospheres 2-4 days after initial seeding into growth media. Representative views of GBM neurospheres are shown in Fig. 1a. Over $60 \%$ of human GBMs with amplified EGFR are known to express the vIII variant of EGFR [3, 9]. We thus examined the protein expression of EGFRvIII for the three GBM lines that we studied: U87-MG, GBM2 and GBM39. GBM2 expressed EGFRvIII levels (Fig. 1b) at an order of magnitude below that of GBM39 (Fig. 1b) while EGFRvIII levels for U87-MG was undetectable (data not shown, [34]).

\section{Dose-dependent inhibition of proliferation by Withaferin A and AshwaMAX-}

We used the alamar blue assay [28] to assess for proliferative activity in U87, GBM2 and GBM39 and the impact on proliferation by both Withaferin A (Fig. 2a) and AshwaMAX. When inhibition studies, (using the alamar blue assay as the readout) were performed with Withaferin A (Fig. 2b), we observed a dose-dependent inhibition of alamar blue activity (and hence proliferative activity) for all three glioblastoma cell lines (U87-MG, GBM2, GBM39). $\mathrm{IC}_{50}$ values were submicromolar and ranged from 0.25 to $0.31 \mu \mathrm{M}$ (Fig. 2b). 
The root extract from the Withania somnifera tree, AshwaMAX, is reported to contain $4.3 \%$ (w/w) Withaferin A [25]. The addition of increasing amounts of AshwaMAX also caused a dosage dependent inhibition of alamar blue activity for U87-MG, GBM2 and GBM39 (Fig. 2c). $\mathrm{IC}_{50}$ values for AshwaMAX extract were $14.8 \mu \mathrm{g} / \mathrm{ml}$ (U87MG), $2.1 \mu \mathrm{g} / \mathrm{ml}$ (GBM2) and $2.4 \mu \mathrm{g} / \mathrm{ml}$ (GBM39). The corresponding $\mathrm{IC}_{50}$ for equivalence of Withaferin A (assuming $4.3 \%$ content of Withaferin A in AshwaMAX) were 1.4, 0.22 and $0.19 \mu \mathrm{M}$ equivalent for U87MG, GBM2 and GBM39 respectively. Thus, in contrast to the Withaferin A alone studies (Fig. 2b), U87 responded with a higher $\mathrm{IC}_{50}$ of inhibition $(14.8 \mu \mathrm{g} / \mathrm{ml})$ than either GBM2 $(2.1 \mu \mathrm{g} / \mathrm{ml})$ or GBM39 $(2.4 \mu \mathrm{g} / \mathrm{ml})$. The findings most likely illustrate the unique environmental, phenotypic and molecular features of U87-MG compared to other patientderived gliomas.

\section{Withaferin A and AshwaMAX affects neurosphere formation and cell viability} of glioblastoma cells specifically-We wished to validate that the abrogation of cell titer blue activity by AshwaMAX or Withferin A is related to a parallel reduction of some cellular function that is implicated to growth and proliferation. When GBM2 was treated with either AshwaMAX $(50 \mathrm{mg} / \mathrm{ml})$ or Withaferin A $(20 \mu \mathrm{M})$, the normal process of neurosphere formation as seen in untreated or DMSO-treated control cells (Fig. 3a) was disrupted as early as $24 \mathrm{~h}$ post-addition(Fig. $3 \mathrm{~b}$, c). Instead, the cultures became largely populated by single cells and any existing neurospheres essentially collapsed (Supplemental Figure S1). Dose-dependent studies on the impact of AshwaMAX in eliminating GBM2 neurosphere formation revealed an $\mathrm{IC}_{50}$ of $2.5 \mu \mathrm{g} / \mathrm{ml}$ (Fig. 3d) a figure that is close to the equivalent $\mathrm{IC}_{50}$ from the alamar blue studies $(2.1 \mu \mathrm{g} / \mathrm{ml})$. Cell viability studies, performed by assessing for the percentage of PI-positive cells, showed that both AshwaMAX and Withaferin A significantly decreased the number of viable cells by nearly four-fold $(n=3, P$ $<0.001$, two-way ANOVA) to less than $20 \% \pm 10$ viable cells (Supplemental Figure S2). There was some cell death brought on by processing and thus cell viability under control, vehicle-treated conditions never reached $100 \%$.

\section{Toxicity studies}

To examine for specificity of inhibition by AshwaMAX and Withaferin A against glioblastoma cells, we did dose response studies for those two drugs on a normal human fibroblast, PCS. These dosage studies were then compared to equivalent experiments performed on U87, GBM2 and GBM39. In general, such investigations revealed a nearly ten-fold greater resistance (as assessed by $\mathrm{IC}_{50}$ ) both to Withaferin $\mathrm{A}(\mathrm{PCS}=2.1, \mathrm{U} 87=$ $0.31, \mathrm{GBM} 2=0.28, \mathrm{GBM} 39=0.25 \mu \mathrm{M})$ and to AshwaMAX $(\mathrm{PCS}=19.62, \mathrm{U} 87=14.8$, GBM2 $=2.1$, GBM39 $=2.4 \mu \mathrm{g} / \mathrm{ml}$ ) when compared to the three glioblastoma lines: U87MG, dGBM2 and GBM39 (Fig. 3e).

\section{Preclinical studies}

\section{Establishment of intra-cranial xenograft with GBM2s expressing GFP/ luciferase fusion protein-As an initial experiment, approximately 400,000} GBM2/gfp/luc cells were injected $3 \mathrm{~mm}$ deep into the right parietal region of the murine brain (Supplemental Figure S3 for equipment set-up). Representative time-courses for changes in BLI with respect to time after implantation are shown in Fig. 4a, c. Initially, there 
is a linear increase in bioluminescent signal from 0 to $\sim 20$ days post-implantation ( $\mathrm{p}<0.02$, four parameter non-linear regression analysis). After 25-30 days, post-implantation, BLI signals stabilized and stayed constant for up to 60 days post-implantation. In fact, several mice have shown stable bioluminescence signal $\left(10^{7}\right.$ to $10^{8}$ photons/sec) for up to 7 months post-implantation. In general, the mice at this time-point had no significant loss in weight or alterations in physical behavior. In the past 7 months, however, about $24 \%$ of GBM2/gfp/ luc-implanted mice showed significant weight loss (31 out of a total of 127 mice) and were subsequently sacrificed. By way of contrast, all mice implanted with U87-MG suffered significant loss of weight and needed to be sacrificed one month post-surgery while mice implanted with GBM39 suffered similar weight loss and needed to be sacrificed at 2 months post-surgery (data not shown).

Time-dependent changes in BLI with oral gavage AshwaMAX-Oral gavages of AshwaMAX (40 mg/kg/day) were given every other day at a point where the BLI signal increased at a linear rate with respect to time (Fig. 4b, c). HPLC analysis of murine plasma confirmed the presence of Withaferin A in the bloodstream with oral gavage treatment of AshwaMAX (Supplemental Figure S5). There was a rapid decline of BLI signal, by nearly an order of magnitude, within 1 week of treatment for all mice. The loss of signal was reproduced in other mice (Fig. 4c) and persisted for nearly 30 days. Indeed there was nearly a complete disappearance of bioluminescence, after-which, the BLI signal recovered and there was a subsequent increase in BLI signal one month after the beginning of AshwaMAX treatment (Fig. 4b, d). Control, vehicle-treated mice showed an increase in BLI signal, followed by a plateau of signal by approximately 30 days after the start of the oral gavages (Fig. 4a, d). During the time of vehicle- versus drug-treatment, no animal suffered a significant loss of weight (Supplemental Figure S6).

\section{Visualization of GBM2/gfp/luc Xenografts by T1-weighted MRI and} bioluminescent microscopy-To test if the glioblastoma xenografts could be visible with a key modality in clinical radiology, mice with intracranial tumors (GBM2 at 28 weeks post-implantation) were subjected to T1-weighted MRI scans in the presence of an intraperitoneally-injected Gd-based contrast agent (Multihance, Bracco S.p.A.). As shown by the sagittal (Fig. 5a) scan, a faint and diffuse MRI signal for GBM2/gfp/luc xenografts appeared at 28 weeks post-implantation. There were no distinctive borders to the MRI signal at that time-point. At the same time, this very same mouse displayed strong BLI signal (Fig. $5 b)$. When sagittal, $30 \mu \mathrm{M}$ frozen sections of brain were prepared, BLI $\backslash$ microscopy imaging revealed a strong but spread-out signal along the sagittal axis and base of the organ (Fig. 5c). $400 \times$ bioluminescent microscopy reveal luciferase activity to be diffused along several tracks of embedded cells within the brain (Fig. 5d).

GBM39 xenografts grew more aggressively than equivalent GBM2 xenografts yet cell culture $\mathrm{IC}_{50}$ s to AshwaMAX were the same, thus we investigated the impact of AshwaMAX treatment (oral gavage, $40 \mathrm{mg} / \mathrm{kg} /$ day) on nude mice with standardized intra-cranial implantations of 400,000 GBM39 cells. Treatment started at 2 weeks post-implantation where BLI scans showed a significant growth in tumor burden. MRI studies showed that, in contrast to GBM2, the GBM39 xenografts for vehicle-treated mice showed distinct regions 
for tumor versus normal brain with several finger-like projections along the corpus callosum and the hippocampus (Supplemental Figure S4A), a pattern that was not detectible by MRI for GBM2/gfp/luc xenografts.

By 7-8 weeks post-implantation, all vehicle-treated mice were dead with total BLI signals above $10^{8}$ photons/sec while AshwaMAX-treated mice survived at least 1 month more (data not shown). When sagittal sections of a vehicle-treated mouse (Supplemental Figure S4B) and an AshwaMAX-treated mouse (Supplemental Figure S4C) were compared (8 weeks post-implantation of xenografts), the AshwaMAX-treated mouse had much diminished total BLIs signal compared to controls but migration of the signal (and hence the cells) was not impaired.

\section{Discussion}

One salient finding arising from the in vitro studies is that AshwaMAX is capable of abrogating the formation and growth of neurospheres in culture. Such results prompted us to perform preclinical experiments that revealed a second salient finding, namely, that oral delivery of a natural product (AshwaMAX) to preclinical models of human glioblastomas (genetically modified to express a GFP/Firefly Luciferase fusion protein) caused an initial diminishment of bioluminescent signal which suggests a reduction in viable numbers of tumor cells. This is consistent with cell culture observations of decreased cellular viability (Supplemental Figure S2) through challenge by AshwaMAX. The eventual recovery of bioluminescence signal suggests that monitoring of gliomas by BLI eventually detected either the expansion of an AshwaMAX-resistant subline or the migration of glioma subpopulations towards microenvironments that are protective against AshwaMAX. Presently, it is unknown as to which process occurs, however, AshwaMAX did not seem to eliminate glioma migration away from the original site of injection (Supplemental Figure S4).

Our study represents the first time that the natural product, AshwaMAX has been shown to be effective in a preclinical model of glioblastoma and that the effectiveness of the drug has been successfully monitored by the molecular modality of bioluminescent imaging. This would indicate that AshwaMAX will be a highly promising candidate for future clinical evaluation. Our studies may also serve as a paradigm on how other natural products indicated for other disorders can be screened as anti-cancer agents. Natural products such as AshwaMAX may potentially be used in conjunction with other drugs such as temozolomide and bevacizumab $[35,36]$ which are established chemotherapies against glioblastoma. A similar research direction could involve combination studies of AshwaMAX with nanoparticle-enhanced radiotherapy (RT) [37] or High-intensity Focused Ultrasound (HIFU) [38]. Such interventions target the brain by breaking down its blood brain barrier and potentially render a previously sheltered tumor accessible to both therapeutic and imaging agents. The steroidal character of Withaferin A confers favorable capacities of the drug to permeate cellular membranes and thus render it capable of penetrating the blood brain barrier. Additional interventions such as RT or HIFU may augment the already good ability of Withaferin A to penetrate and cross the barrier. 
Optimizing therapeutic effectiveness of AshwaMAX may also involve a deeper study on the effective therapeutic window of AshwaMAX. For instance, the steroidal character of Withaferin A as well as other compounds in AshwaMAX may have an immunosuppressive effect on the patient. This has implications in examining future applications of AshwaMAX and Withaferin A in combination with immunotherapy protocols. Thus AshwaMAX's impact on the tumor microenvironment of gliomas will need to be investigated, first in murine models that are fully immunocompetent and eventually in humans receiving immunotherapies.

In the entire AshwaMAX study, the mice suffered no loss of weight (Supplemental Figure S6). This finding is congruent with reports in the literature showing a general lack of toxicity with treatment by similar or same formulations of winter cherry tree extracts [11, 39-41] in humans. The research results, in aggregate, strengthens the validity in utilizing a natural plant extract such as AshwaMAX to treat gliomas.

AshwaMAX has advantages to the pure Withaferin A partly because animal studies with pure drug is more costly than with the natural product. This may confer insights on clinical costs for future human applications. More importantly, the $\mathrm{IC}_{50}$ equivalent dose for Withaferin A tend to be lower for AshwaMAX in GBM2 and GBM39 than the comparable $\mathrm{IC}_{50} \mathrm{~s}$ for purified Withaferin A (Fig. 2b). Our experiments thus infer that the presence of compounds other than withanolides may aide AshwaMAX to be a more potent therapeutic than Withaferin A alone although more research will be required to confirm this.

In order to track the effectiveness of AshwaMAX therapy in a clinical setting, one must have a convenient modality to monitor glioma tumors in the brain and therefore have a practical way to monitor therapeutic response. Magnetic resonance imaging (MRI) is the most common modality of choice; however, such an approach is expensive, the procedure tends to be prolonged and laborious for the patient, and visualization may not be apparent if the tumor grows diffusely in the brain [25]. Indeed examination of GBM2/gfp/Luc xenografts via bioluminescent microscopy would suggest that diffuse growth and migration is characteristic of the cancer. Thus it is unlikely that MRI alone will be useful in tracking its progress and its responsiveness to therapy.

Optimizing therapeutic usefulness of AshwaMAX and by implication, Withanolides, will be aided by further investigations into the mechanism of action of Withaferin A. Although comprehensive mechanistic information explaining Withaferin A's anti-tumoral properties remains lacking, there are provocative suggestions in the literature on how the lactonal steroid actually works. Reports strongly indicate that Withaferin A can affect the cytoskeletal organization [42, 43] as well as proteasomal activation [44] in tumor cells. Growth stimulation, angiogenesis induction by tumor cells as well as susceptibility to apoptosis are all impacted with Withaferin A potentially through the effect of the steroid on the expression of activity of heat shock proteins and the transcription factor, NF- $\mathrm{kB}$ [45]. Withaferin A may exert its effect (either directly or through its modulation of heat shock protein 90) on several kinases such as PKC, MAP Kinases, P38 and JNK, AK+ and ERK [46-48]. As a consequence, future studies will focus upon elucidating details of mechanisms and thereby gaining insights on how to fine-tune the effectiveness of Withaferin A. 
Future directions to elucidate mechanisms will include the study of more glioma lines exhibiting differential expression of key molecular markers such as EGFR and EGFRvIII but also FGF and PDGF. We can thus probe how generalizable is the therapeutic effectiveness of AshwaMAX and Withaferin A. The relationship between intrinsic diffusiveness of GBM lines in xenografts and sensitivity to drugs such as Withaferin A or AshwaMAX should also be elucidated. In order to maximize effectiveness of anti-glioblastoma drugs, circulating biomarkers whose presence indicate the onset of resistant sub clones ought to be identified and characterized. The newly discovered lymphatic circulation system that resides within the dural sinuses of the central nervous system [49] may also contribute to the accessibility of drugs (and maybe their metabolism) within the brain. Such research should improve the clinical utility of the natural product, AshwaMAX and by implication, Withaferin A.

\section{Supplementary Material}

Refer to Web version on PubMed Central for supplementary material.

\section{Acknowledgments}

GBM39 was the donated to us by Dr. Paul Mischel, MD, PhD while the cell lines of GBM2 and GBM2/gfp/Luc were kindly provided to us by Dr. Michelle Monje, MD, PhD. We thank Dr's. Laura Pisani, PhD and Timothy Doyle, PhD of the Stanford Small Animal Imaging Facility (SAIF) for their excellent technical support. AshwaMAX was obtained from Dr. Lal Hingorani from Pharmaza Herbal Pvt Ltd. (Gujarat, India). We acknowledge the excellent technical support of Kathryn Li, Tara Thakurta, Alex Serafini and Xiaofan Wu.

Funding

We gratefully acknowledge the Ben and Catherine Ivy Foundation for funding of the research.

\section{Abbreviations}

$\begin{array}{ll}\text { BLI } & \text { Bioluminescence Imaging } \\ \text { DAPI } & 4^{\prime}, 6 \text {-Diamidino-2-Phenylindole } \\ \text { h-EGF } & \text { Human Epidermal Growth Factor } \\ \text { EGFR } & \text { Epidermal Growth Factor Receptor } \\ \text { EGFRvIII } & \text { Epidermal Growth Factor Receptor variant III } \\ \text { h-FGF } & \text { Human Fibroblast Growth Factor } \\ \text { GBM } & \text { Glioblastoma Multiforme } \\ \text { GBM2 } & \begin{array}{l}\text { Patient-derived glioblastoma multiforme cell culture (from } \\ \text { Stanford University School of Medicine) }\end{array} \\ \text { GBM2/gfp/luc } & \begin{array}{l}\text { GBM2 that is genetically modified to express a fusion } \\ \text { protein of firefly luciferase and Green Fluorescence Protein } \\ \text { (GFP) }\end{array} \\ \text { GBM39 } & \text { Patient-derived glioblastoma multiforme cell culture (from } \\ \text { University of California at San Diego School of Medicine) }\end{array}$




$\begin{array}{ll}\text { GFP } & \text { Green Fluorescent Protein } \\ \text { GFP/Luc } & \begin{array}{l}\text { Fusion protein of Green Fluorescence Protein and firefly } \\ \text { luciferase }\end{array} \\ \text { HSP70 } & \text { Heat Shock Protein } 70 \\ \text { PBS } & \text { Phosphate Buffered Saline } \\ \text { h-PDGF-AA } & \text { Human Platelet-derived Growth Factor variant AA } \\ \text { h-PDGF-BB } & \text { Human Platelet-derived Growth Factor variant BB } \\ \text { U87 } & \text { GBM cell line purchased from ATCC }\end{array}$

\section{References}

1. Johnson DR, O'Neill BP. Glioblastoma survival in the United States before and during the temozolomide era. J Neurooncol. 2012; 107:359-364. DOI: 10.1007/s11060-011-0749-4 [PubMed: 22045118]

2. Vanden Berghe W, Sabbe L, Kaileh M, Haegeman G, Heyninck K. Molecular insight in the multifunctional activities of Withaferin A. Biochem Pharmacol. 2012; 84:1282-1291. DOI: 10.1016/j.bcp.2012.08.027 [PubMed: 22981382]

3. Thakkar JP, Dolecek TA, Horbinski C, Ostrom QT, Lightner DD, Barnholtz-Sloan JS, Villano JL. Epidemiologic and molecular prognostic review of glioblastoma. Cancer Epidemiol Biomark Prev. 2014; 23:1985-1996. DOI: 10.1158/1055-9965.EPI-14-0275

4. Le Mercier M, Hastir D, Moles Lopez X, De Neve N, Maris C, Trepant AL, Rorive S, Decaestecker C, Salmon I. A simplified approach for the molecular classification of glioblastomas. PLoS ONE. 2012; 7:e45475.doi: 10.1371/journal.pone.0045475 [PubMed: 23029035]

5. Cloughesy TF, Cavenee WK, Mischel PS. Glioblastoma: from molecular pathology to targeted treatment. Annu Rev Pathol. 2014; 9:1-25. DOI: 10.1146/annurev-pathol-011110-130324 [PubMed: 23937436]

6. Verhaak RG, Hoadley KA, Purdom E, Wang V, Qi Y, Wilkerson MD, Miller CR, Ding L, Golub T, Mesirov JP, Alexe G, Lawrence M, O'Kelly M, Tamayo P, Weir BA, Gabriel S, Winckler W, Gupta S, Jakkula L, Feiler HS, Hodgson JG, James CD, Sarkaria JN, Brennan C, Kahn A, Spellman PT, Wilson RK, Speed TP, Gray JW, Meyerson M, Getz G, Perou CM, Hayes DN. Integrated genomic analysis identifies clinically relevant subtypes of glioblastoma characterized by abnormalities in PDGFRA, IDH1, EGFR, and NF1. Cancer Cell. 2010; 17:98-110. DOI: 10.1016/j.ccr.2009.12.020 [PubMed: 20129251]

7. Zhang X, Zhang W, Cao WD, Cheng G, Zhang YQ. Glioblastoma multiforme: molecular characterization and current treatment strategy (Review). Exp Ther Med. 2012; 3:9-14. DOI: 10.3892/etm.2011.367 [PubMed: 22969836]

8. Olar A, Aldape KD. Using the molecular classification of glioblastoma to inform personalized treatment. J Pathol. 2014; 232:165-177. DOI: 10.1002/path.4282 [PubMed: 24114756]

9. Frederick L, Wang XY, Eley G, James CD. Diversity and frequency of epidermal growth factor receptor mutations in human glioblastomas. Cancer Res. 2000; 60:1383-1387. [PubMed: 10728703]

10. Grasso CS, Tang Y, Truffaux N, Berlow NE, Liu L, Debily MA, Quist MJ, Davis LE, Huang EC, Woo PJ, Ponnuswami A, Chen S, Johung TB, Sun W, Kogiso M, Du Y, Qi L, Huang Y, HuttCabezas M, Warren KE, Le Dret L, Meltzer PS, Mao H, Quezado M, van Vuurden DG, Abraham J, Fouladi M, Svalina MN, Wang N, Hawkins C, Nazarian J, Alonso MM, Raabe EH, Hulleman E, Spellman PT, Li XN, Keller C, Pal R, Grill J, Monje M. Functionally defined therapeutic targets in diffuse intrinsic pontine glioma. Nat Med. 2015; doi: 10.1038/nm.3855

11. Mishra LC, Singh BB, Dagenais S. Scientific basis for the therapeutic use of Withania somnifera (ashwagandha): a review. Alternative Med Rev. 2000; 5:334-346. 
12. Weir SJ, DeGennaro LJ, Austin CP. Repurposing approved and abandoned drugs for the treatment and prevention of cancer through public-private partnership. Cancer Res. 2012; 72:1055-1058. DOI: 10.1158/0008-5472.CAN-11-3439 [PubMed: 22246671]

13. Pantziarka P, Bouche G, Meheus L, Sukhatme V, Sukhatme VP. Repurposing drugs in oncology (ReDO)-mebendazole as an anti-cancer agent. Ecancermedicalscience. 2014; 8:443.doi: 10.3332/ ecancer.2014.443 [PubMed: 25075217]

14. Lavie D, Glotter E, Shvo Y. Constituents of Withania somnifera Dun. III. The side chain of Withaferin A. J Org Chem. 1965; 30:1774-1778.

15. Mirjalili MH, Moyano E, Bonfill M, Cusido RM, Palazon J. Steroidal lactones from Withania somnifera, an ancient plant for novel medicine. Molecules. 2009; 14:2373-2393. DOI: 10.3390/ molecules14072373 [PubMed: 19633611]

16. Prasanna KS, Shilpa P, Salimath BP. Withaferin A suppresses the expression of vascular endothelial growth factor in Ehrlich ascites tumor cells via Sp1 transcription factor. Curr Trends Biotechnol Pharm. 2009; 3:138-148.

17. Heyninck K, Lahtela-Kakkonen M, Van der Veken P, Haegeman G, Vanden Berghe W. Withaferin A inhibits NF-kappaB activation by targeting cysteine 179 in IKKbeta. Biochem Pharmacol. 2014; 91:501-509. DOI: 10.1016/j.bcp.2014.08.004 [PubMed: 25159986]

18. Khan S, Rammeloo AW, Heikkila JJ. Withaferin A induces proteasome inhibition, endoplasmic reticulum stress, the heat shock response and acquisition of thermotolerance. PLoS ONE. 2012; 7:e50547.doi: 10.1371/journal.pone.0050547 [PubMed: 23226310]

19. Jilani K, Lupescu A, Zbidah M, Shaik N, Lang F. Withaferin A-stimulated $\mathrm{Ca}^{2+}$ entry, ceramide formation and suicidal death of erythrocytes. Toxicol Vitro. 2013; 27:52-58. DOI: 10.1016/j.tiv. 2012.09.004

20. Sharada AC, Solomon FE, Devi PU, Udupa N, Srinivasan KK. Antitumor and radiosensitizing effects of withaferin A on mouse Ehrlich ascites carcinoma in vivo. Acta Oncol. 1996; 35:95-100. [PubMed: 8619948]

21. Devi PU, Sharada AC, Solomon FE. Antitumor and radiosensitizing effects of Withania somnifera (Ashwagandha) on a transplantable mouse tumor, Sarcoma-180. Indian J Exp Biol. 1993; 31:607611. [PubMed: 8225418]

22. Widodo N, Kaur K, Shrestha BG, Takagi Y, Ishii T, Wadhwa R, Kaul SC. Selective killing of cancer cells by leaf extract of Ashwagandha: identification of a tumor-inhibitory factor and the first molecular insights to its effect. Clin Cancer Res. 2007; 13:2298-2306. DOI: 10.1158/1078-0432.CCR-06-0948 [PubMed: 17404115]

23. Shohat B, Gitter S, Lavie D. Effect of withaferin A on Ehrlich ascites tumor cells-cytological observations. Int J Cancer. 1970; 5:244-252. [PubMed: 5449164]

24. Shohat B, Gitter S, Abraham A, Lavie D. Antitumor activity of withaferin A (NSC-101088). Cancer Chemother Rep Part. 1967; 1(51):271-276.

25. Patel S, Rao NHL. Safety assessment of Withania Somnifera extract standardized for Withaferin A: acute and Sub-acute toxicity study. 2014

26. Sarkaria JN, Yang L, Grogan PT, Kitange GJ, Carlson BL, Schroeder MA, Galanis E, Giannini C, $\mathrm{Wu}$ W, Dinca EB, James CD. Identification of molecular characteristics correlated with glioblastoma sensitivity to EGFR kinase inhibition through use of an intracranial xenograft test panel. Mol Cancer Ther. 2007; 6:1167-1174. DOI: 10.1158/1535-7163.MCT-06-0691 [PubMed: 17363510]

27. Sasportas LS, Gambhir SS. Imaging circulating tumor cells in freely moving awake small animals using a miniaturized intravital microscope. PLoS ONE. 2014; 9:e86759.doi: 10.1371/journal.pone. 0086759 [PubMed: 24497977]

28. Anoopkumar-Dukie S, Carey JB, Conere T, O’Sullivan E, van Pelt FN, Allshire A. Resazurin assay of radiation response in cultured cells. Br J Radiol. 2005; 78:945-947. DOI: 10.1259/bjr/54004230 [PubMed: 16177019]

29. Lecoeur H. Nuclear apoptosis detection by flow cytometry: influence of endogenous endonucleases. Exp Cell Res. 2002; 277:1-14. DOI: 10.1006/excr.2002.5537 [PubMed: 12061813] 
30. Yoshimoto K, Dang J, Zhu S, Nathanson D, Huang T, Dumont R, Seligson DB, Yong WH, Xiong Z, Rao N, Winther H, Chakravarti A, Bigner DD, Mellinghoff IK, Horvath S, Cavenee WK, Cloughesy TF, Mischel PS. Development of a real-time RT-PCR assay for detecting EGFRvIII in glioblastoma samples. Clin Cancer Res. 2008; 14:488-493. DOI: 10.1158/1078-0432.CCR-07-1966 [PubMed: 18223223]

31. Baumann BC, Dorsey JF, Benci JL, Joh DY, Kao GD. Stereotactic intracranial implantation and in vivo bioluminescent imaging of tumor xenografts in a mouse model system of glioblastoma multiforme. J Vis Exp. 2012; doi: 10.3791/4089

32. Bergamino M, Bonzano L, Levrero F, Mancardi GL, Roccatagliata L. A review of technical aspects of T1-weighted dynamic contrast-enhanced magnetic resonance imaging (DCE-MRI) in human brain tumors. Phys Med. 2014; 30:635-643. DOI: 10.1016/j.ejmp.2014.04.005 [PubMed: 24793824]

33. Llewellyn BD. Nuclear staining with alum hematoxylin. Biotech Histochem. 2009; 84:159-177. DOI: 10.1080/10520290903052899 [PubMed: 19579146]

34. Del Vecchio CA, Giacomini CP, Vogel H, Jensen KC, Florio T, Merlo A, Pollack JR, Wong AJ. EGFRvIII gene rearrangement is an early event in glioblastoma tumorigenesis and expression defines a hierarchy modulated by epigenetic mechanisms. Oncogene. 2013; 32:2670-2681. DOI: 10.1038/onc.2012.280 [PubMed: 22797070]

35. Sepulveda JM, Belda-Iniesta C, Gil-Gil M, Perez-Segura P, Berrocal A, Reynes G, Gallego O, Capellades J, Ordonez JM, La Orden B, Balana C. A phase II study of feasibility and toxicity of bevacizumab in combination with temozolomide in patients with recurrent glioblastoma. Clin Transl Oncol. 2015; doi: 10.1007/s12094-015-1304-0

36. Johansson F, Ekman S, Blomquist E, Henriksson R, Bergstrom S, Bergqvist M. A review of dosedense temozolomide alone and in combination with bevacizumab in patients with first relapse of glioblastoma. Anticancer Res. 2012; 32:4001-4006. [PubMed: 22993350]

37. Joh DY, Sun L, Stangl M, Al Zaki A, Murty S, Santoiemma PP, Davis JJ, Baumann BC, AlonsoBasanta M, Bhang D, Kao GD, Tsourkas A, Dorsey JF. Selective targeting of brain tumors with gold nanoparticle-induced radiosensitization. PLoS ONE. 2013; 8:e62425.doi: 10.1371/ journal.pone.0062425 [PubMed: 23638079]

38. Coluccia D, Fandino J, Schwyzer L, O’Gorman R, Remonda L, Anon J, Martin E, Werner B. First noninvasive thermal ablation of a brain tumor with MR-guided focused ultrasound. J Ther Ultrasound. 2014; 2:17.doi: 10.1186/2050-5736-2-17 [PubMed: 25671132]

39. Raut AA, Rege NN, Tadvi FM, Solanki PV, Kene KR, Shirolkar SG, Pandey SN, Vaidya RA, Vaidya AB. Exploratory study to evaluate tolerability, safety, and activity of Ashwagandha (Withania somnifera) in healthy volunteers. J Ayurveda Integr Med. 2012; 3:111-114. DOI: 10.4103/0975-9476.100168 [PubMed: 23125505]

40. Wadhwa R, Singh R, Gao R, Shah N, Widodo N, Nakamoto T, Ishida Y, Terao K, Kaul SC. Water extract of Ashwagandha leaves has anticancer activity: identification of an active component and its mechanism of action. PLoS ONE. 2013; 8:e77189.doi: 10.1371/journal.pone.0077189 [PubMed: 24130852]

41. Sandhu JS, Shah B, Shenoy S, Chauhan S, Lavekar GS, Padhi MM. Effects of Withania somnifera (Ashwagandha) and Terminalia arjuna (Arjuna) on physical performance and cardiorespiratory endurance in healthy young adults. Int J Ayurveda Res. 2010; 1:144-149. DOI: 10.4103/0974-7788.72485 [PubMed: 21170205]

42. Satelli A, Li S. Vimentin in cancer and its potential as a molecular target for cancer therapy. Cell Mol Life Sci. 2011; 68:3033-3046. DOI: 10.1007/s00018-011-0735-1 [PubMed: 21637948]

43. Bargagna-Mohan P, Hamza A, Kim YE, Khuan Abby Ho Y, Mor-Vaknin N, Wendschlag N, Liu J, Evans RM, Markovitz DM, Zhan CG, Kim KB, Mohan R. The tumor inhibitor and antiangiogenic agent withaferin A targets the intermediate filament protein vimentin. Chem Biol. 2007; 14:623634. DOI: 10.1016/j.chembiol.2007.04.010 [PubMed: 17584610]

44. Yang H, Shi G, Dou QP. The tumor proteasome is a primary target for the natural anticancer compound Withaferin A isolated from "Indian winter cherry". Mol Pharmacol. 2007; 71:426-437. DOI: 10.1124/mol.106.030015 [PubMed: 17093135]

45. Grover A, Shandilya A, Punetha A, Bisaria VS, Sundar D. Inhibition of the NEMO/IKKbeta association complex formation, a novel mechanism associated with the NF-kappaB activation 
suppression by Withania somnifera's key metabolite withaferin A. BMC Genom. 2010; 11(Suppl 4):S25.doi: 10.1186/1471-2164-11-S4-S25

46. Yu Y, Hamza A, Zhang T, Gu M, Zou P, Newman B, Li Y, Gunatilaka AA, Zhan CG, Sun D. Withaferin A targets heat shock protein 90 in pancreatic cancer cells. Biochem Pharmacol. 2010; 79:542-551. DOI: 10.1016/j.bcp.2009.09.017 [PubMed: 19769945]

47. Grover A, Shandilya A, Agrawal V, Pratik P, Bhasme D, Bisaria VS, Sundar D. Hsp90/Cdc37 chaperone/co-chaperone complex, a novel junction anticancer target elucidated by the mode of action of herbal drug Withaferin A. BMC Bioinformatics. 2011; 12(Suppl 1):S30.doi: 10.1186/1471-2105-12-S1-S30 [PubMed: 21342561]

48. Sen N, Banerjee B, Das BB, Ganguly A, Sen T, Pramanik S, Mukhopadhyay S, Majumder HK. Apoptosis is induced in leishmanial cells by a novel protein kinase inhibitor withaferin $\mathrm{A}$ and is facilitated by apoptotic topoisomerase I-DNA complex. Cell Death Differ. 2007; 14:358-367. DOI: 10.1038/sj.cdd.4402002 [PubMed: 16841091]

49. Louveau A, Smirnov I, Keyes TJ, Eccles JD, Rouhani SJ, Peske JD, Derecki NC, Castle D, Mandell JW, Lee KS, Harris TH, Kipnis J. Structural and functional features of central nervous system lymphatic vessels. Nature. 2015; doi: 10.1038/nature14432 
Brightfield Microscopy
(10X Magnification)

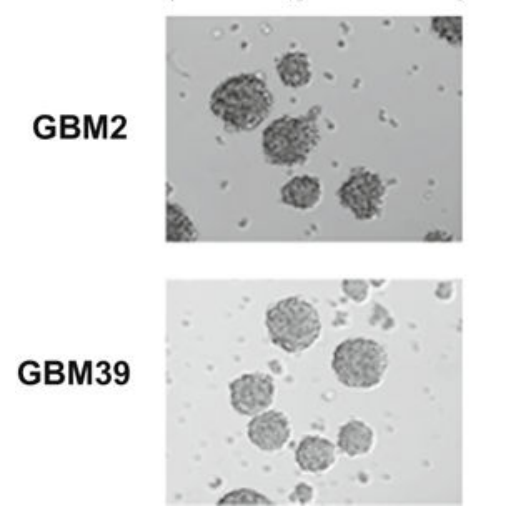

b Western Blot: EGFR vIII

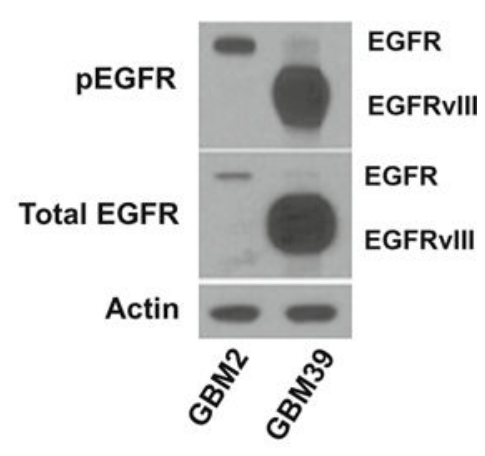

Fig. 1.

Morphological and molecular characterization of glioblastoma cell lines GBM39 and patient-derived GBM2. Cell line 1 = GBM2, Cell line 2 = GBM39. a Bright field microscopy of human glioblastoma cell lines. b Western blot for antibodies to EGFRvIII (total and phosphorylated) in GBM2 and GBM39. Control is beta-actin. U87-MG expressed virtually no EGFRvIII 
a Withaferin A

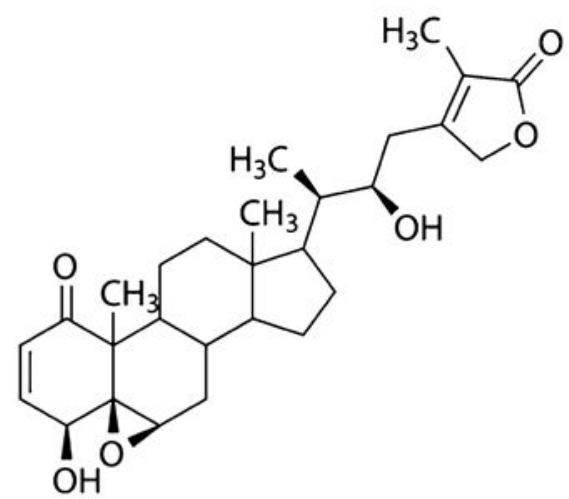

b Withaferin A

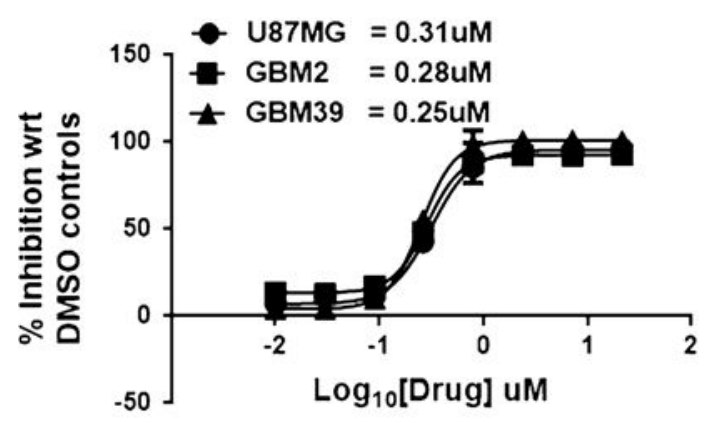

c AshwaMAX

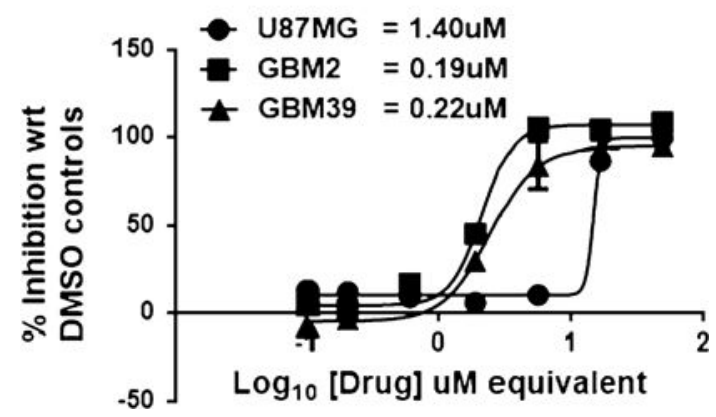

Fig. 2.

a Chemical structure of Withaferin A. Dose-dependent inhibition of cellular proliferation by b Withaferin A and c AshwaMAX on GBM39, GBM2) and U87-MG. For Withaferin A, $\mathrm{IC}_{50}$ 's were at $0.31 \mu \mathrm{M}$ (U87MG), $0.28 \mu \mathrm{M}$ (GBM2) and $0.25 \mu \mathrm{M}$ (GBM39). For AshwaMAX, IC 50 's were at $14.8 \mu \mathrm{g} / \mathrm{ml}$ (U87MG), $2.1 \mu \mathrm{g} / \mathrm{ml}$ (GBM2) and $2.4 \mu \mathrm{g} / \mathrm{ml}$ (GBM39) or, if assuming $4.3 \%$ Withaferin A in AshwaMAX: 1.4, 0.22 and $0.19 \mu \mathrm{M}$ equivalent respectively. All measurements are done in triplicate 
a

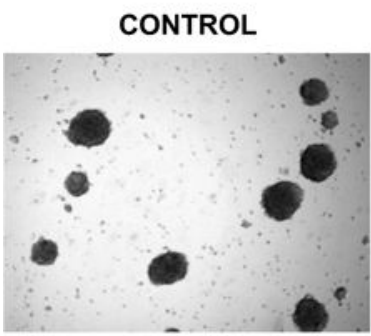

b

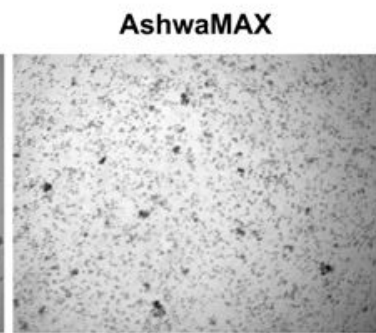

c

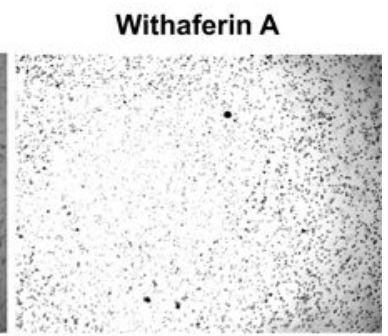

d Effect of AshwamaX on Neurosphere Formation in pcGBM2
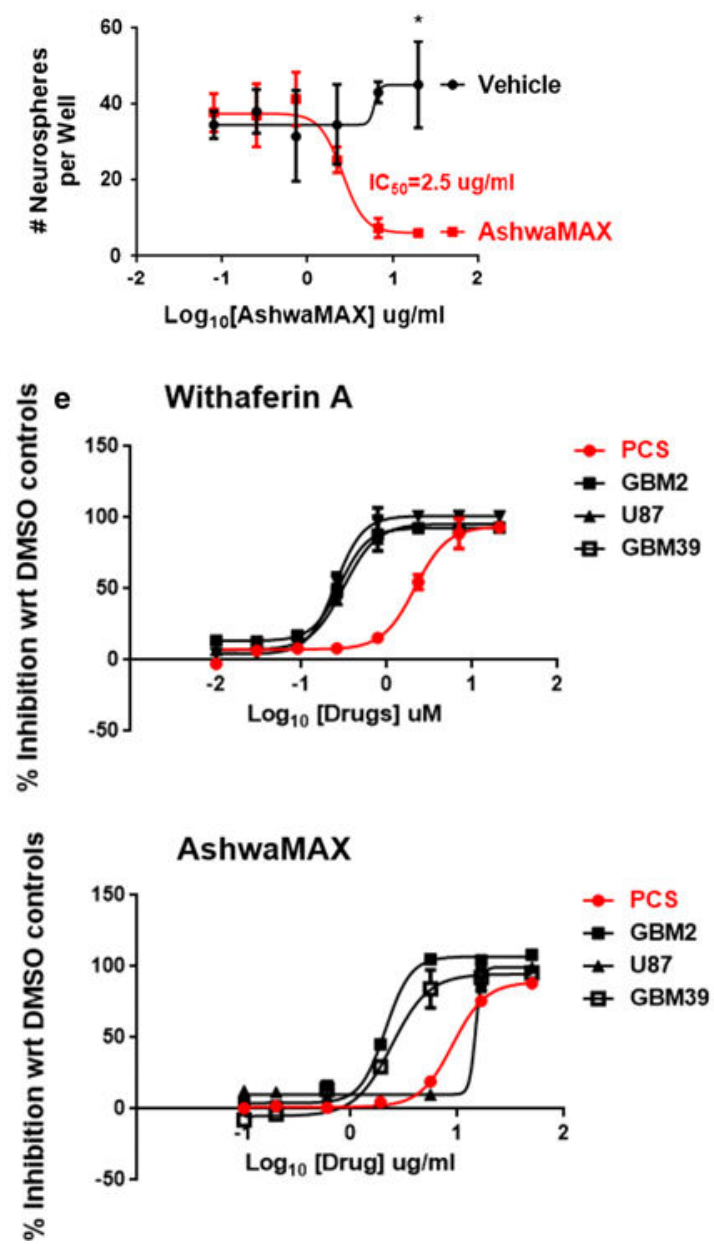

Fig. 3.

$\times 10$ Bright field microscopy of either a DMSO (0.1\%)-treated GBM2 or GBM2 that are challenged with b $50 \mu \mathrm{g} / \mathrm{ml}$ AshwaMAX or c $20 \mu \mathrm{M}$ Withaferin A for 3 days. d Corresponding neurosphere formation studies for samples of GBM2 treated with $0.1 \%$ DMSO (Vehicle Control_black graph, round black data pints) or with $50 \mu \mathrm{g} / \mathrm{ml}$ AshwaMAX (red graph, square, red data points) for 3 days. ${ }^{*} \mathrm{p}<0.002$ control vs. treatment for $n=3$ experiments per group. Neurosphere numbers counted automatically by Celigo $S$ Automatic Cell Cytometer. e Comparative dose-inhibition studies for the drugs Withaferin A 
and AshwaMAX of PCS versus the human glioblastoma lines: U87, GBM2 and GBM39. For Withaferin $\mathrm{A}$ the $\mathrm{IC}_{50} \mathrm{~s}$ are: $\mathrm{PCS}=2.1, \mathrm{U} 87=0.31, \mathrm{GBM} 2=0.28, \mathrm{GBM} 39=0.25 \mu \mathrm{M}$ and for AshwaMAX, the $\mathrm{IC}_{50}$ s are: $\mathrm{PCS}=19.62, \mathrm{U} 87=14.8, \mathrm{GBM} 2=2.1, \mathrm{GBM} 39=2.4$ $\mu \mathrm{g} / \mathrm{ml}$. All measurements are done in triplicate 

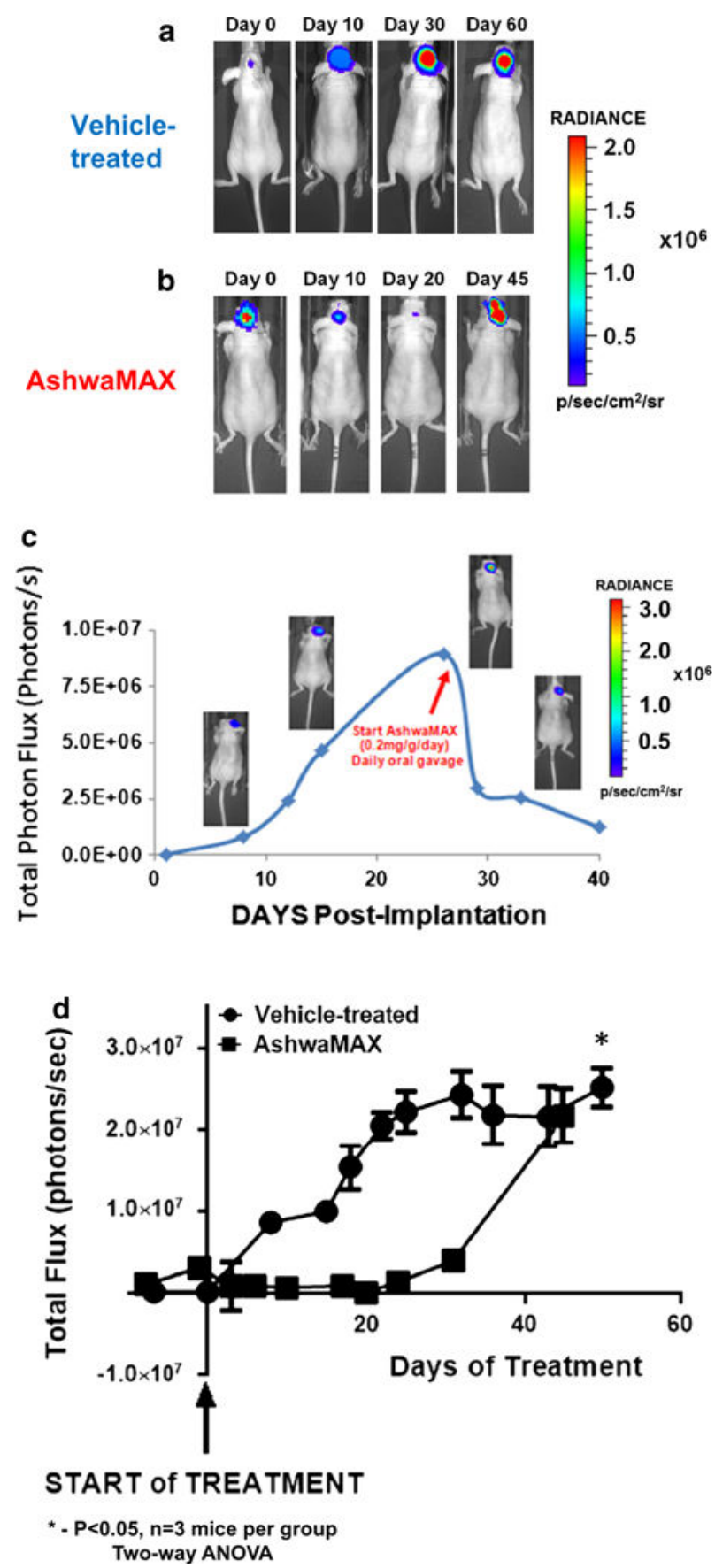

Fig. 4.

Preclinical examination of the influence of AshwaMAX on growing intra-cranial tumors of gfp/luciferase-expressing GBM2. Representative BLI scans for mice with orthotopic xenografts of GBM2 expressing GFP/Luciferase that are either a vehicle-treated or $\mathbf{b}$ given oral gavage, every second day, of AshwaMAX ( $40 \mathrm{mg} / \mathrm{kg} / \mathrm{day}$ ). c Representative, BLI profile of mice with an orthotopic GBM2/GFP/Luc cranial implant when given oral gavage, every other day, of AshwaMAX ( $40 \mathrm{mg} / \mathrm{kg} / \mathrm{day}$ ). d Representative graphical quantification of murine BLI for control, vehicle-treated mice (round data points) and mice given oral gavage 
every other day of AshwaMAX (square data points) at $40 \mathrm{mg} / \mathrm{kg} /$ day ( $\mathrm{n}=3$ mice per experiment, slope of plot significantly different from zero $(\mathrm{P}<0.02)$ as assessed via four parameter non-linear regression analysis). Plot for AshwaMAX treatment compared to vehicle-treatment is significantly different at $\mathrm{P}<0.05$ for $\mathrm{n}=3$ mice per group as assessed via two-way ANOVA 


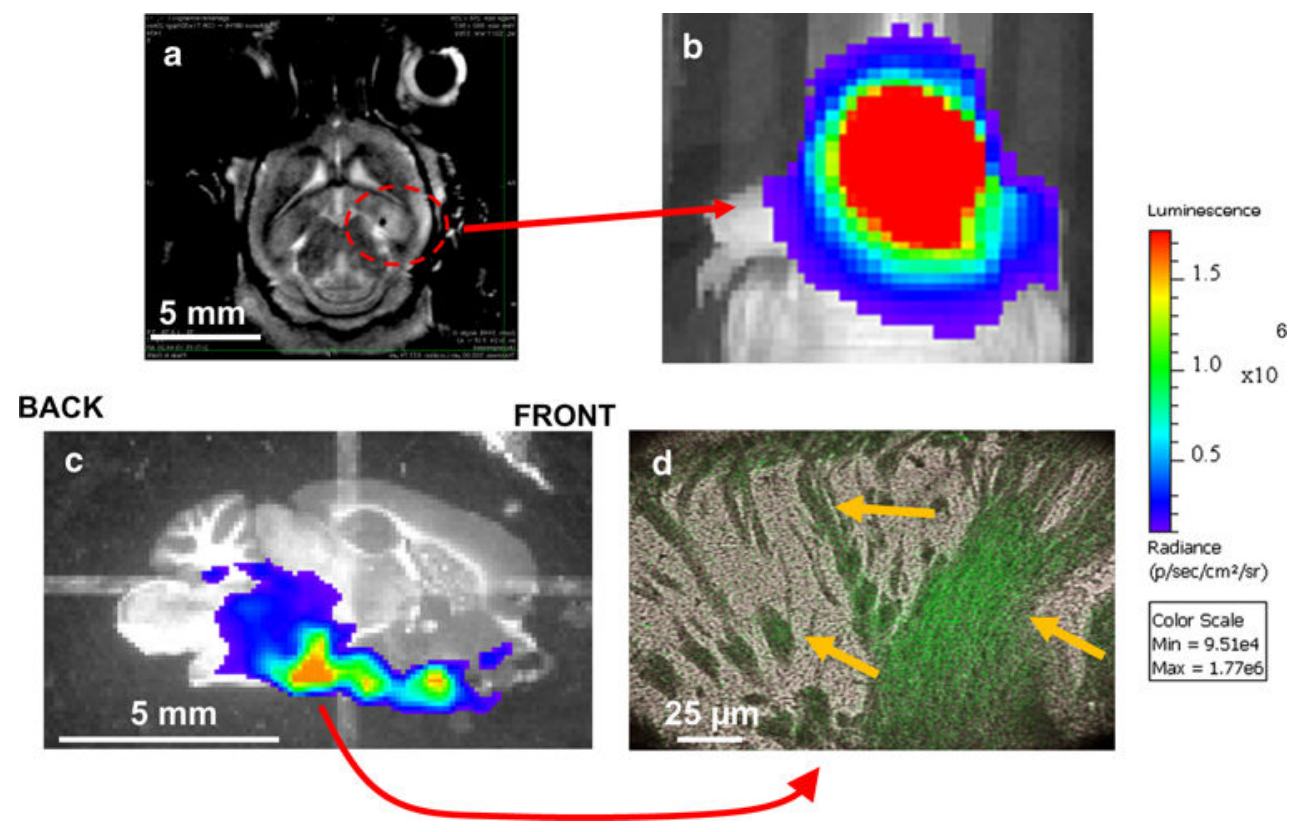

Fig. 5.

a Coronal T1-weighted MRI scans for GBM2/GFP/LUC orthotopic implants at 28 weeks.

Contrast agent was Multihance. b Corresponding, preclinical BLI scan of a nude mice with a 6 month orthotopic implantation of GBM2/gfp/luc. Site of implantation of cell line was 3 $\mathrm{mm}$ deep into the parietal cortical region of the right hemisphere, about $2 \mathrm{~mm}$ to the right and $0.5 \mathrm{~mm}$ anterior to the lamda suture. $30 \mathrm{~s}$ acquisition scan for bioluminescent image, $1 \times$ 1 binning and F-stop of 1 . c BLI scan of a $30 \mu \mathrm{M}$ sagittal section of the right side of the murine brain. One second acquisition scan with standard $1 \times 1$ binning and F-stop of 1 . Dynamic range shown as $6.2 \times 10^{8}$ to $1.9 \times 10^{9} \mathrm{p} / \mathrm{s} / \mathrm{cm}^{2} / \mathrm{sr}$. d Representative example of a $400 \times$ bioluminescent microscopic image for a BLI-positive region from the tissue section shown in "C". Cellular morphology is shown in brightfield while bioluminescent activity is shown in false-color as green. 10 min acquisition time with Olympus LV200 bioluminescent microscope. White bars represent size scale of each pictograph. Yellow arrows indicate individual cells and structures that are also positive for bioluminescent activity 